\title{
Delayed optical images \\ through coupled-resonator-induced transparency
}

\author{
Parvin Sultana $^{1}$, Akira Takami ${ }^{1}$, Takahiro Matsumoto ${ }^{2}$, and Makoto Tomita ${ }^{1}$ \\ ${ }^{1}$ Department of Physics, Faculty of Science, Shizuoka University, \\ 836 Ohya, Suruga-ku, Shizuoka, Japan 422-8529 \\ ${ }^{2}$ Research and Development Center, Stanley Electric Corporation, 5-9-5 \\ Tokodai, Tsukuba, Japan 300-2635
}

\begin{abstract}
Transverse two-dimensional images encoded on optical pulses are propagated through a frequency window of coupled-image-resonator-induced transparency. The optical images are stored and delayed by $10.6 \mathrm{~ns}$, reflecting the tunable dispersion of the coupled resonator. The $k$-space bandwidth of the amplitude transfer function of the system is discussed in the presence of the offresonance Fano interference effect between the two resonators.
\end{abstract}


With rapidly developing technologies required for practical implementation, coupled resonators have been attracting increasing attention for photonic devices, including optical delay lines, buffering, optical networks, rotation sensors, and optical computing schemes [1-7]. Specifically, slow light propagation in coupled resonator system is under intensive investigation to achieve large and tunable delay time, wide bandwidth, preservation of phase information and fast reconfigurable structures [2]. It is a challenging problem to extend the slow light technique in coupled resonator systems into two-dimensional image propagation, because all-optical methods for delaying images have great potential in many applications, including holography and optical pattern correlation measurements, or futuristic all-optical image router for large capacity image information. As a candidate to realize the delayed and stored image, coherently controlled quantum states in atoms, specifically, electromagnetic induced transparency (EIT) is a possible system [8-10]. Such atomic systems are, however, severely constrained to operate at only particular wavelength and have very limited bandwidth; hence, all-optical slow image propagation based on photonic structural dispersion is highly desired. A critical issue for coupled image resonator is, whether it is possible to transmit two-dimensional images through the coupled image resonator, avoiding the effect of diffraction. Here, we experimentally demonstrate that transverse two-dimensional images encoded on optical pulses can be propagated and delayed through a coupled image resonator which represents an alloptical analogue of atomic EIT.

Figure 1 shows a schematic illustration of the experimental setup. For the propagation of twodimensional optical images, a parallel processing system is required. We employed a tandem pair of Fabry-Perot cavities as a coupled image resonator, consisting of three mirrors with reflectivity $R_{i}=$ $r_{i} r_{i}^{*}=r_{i}^{\prime} r_{i}^{\prime *}$, and transmittivity $T_{i}=t_{i} t_{i}{ }^{*}=t_{i}^{\prime} t_{i}{ }^{*}$, where $i=1,2,3$ for the first, second, and third mirrors, respectively. Comparing with the single Fabry-Perot resonator [11], this system has the additional third mirror to compose a coupled resonator, which makes the function more interesting and useful. The amplitude transfer function of the coupled image resonator, $T\left(k_{x}, k_{y}, v\right)$ is given as 


$$
T\left(k_{x}, k_{y}, v\right)=\left\{r_{1}-\frac{t_{1} t_{1}{ }^{\prime} S_{2} e^{-2 \rho_{1}+2 i \phi_{1}}}{1-r_{1}{ }^{\prime} S_{2} e^{-2 \rho_{1}+2 i \phi_{1}}}\right\},
$$

here

$$
S_{2}\left(k_{x}, k_{y}, v\right)=r_{2}-\frac{t_{2} t_{2}{ }^{\prime} r_{3} e^{-2 \rho_{2}+2 i \phi_{2}}}{1-r_{2}{ }^{\prime} r_{3} e^{-2 \rho_{2}+2 i \phi_{2}}},
$$

where $\phi_{j}\left(k_{x}, k_{y}, v\right)=-i L_{j}\left(k_{0}^{2}-k_{x}^{2}-k_{y}^{2}\right)^{1 / 2}, j=1,2$ for the first and second cavities, respectively; $k_{0}=2 \pi v / c, c$ is the velocity of light, and $v$ is the frequency of the laser and $\rho_{1}$, $\rho_{2}$ are the losses in the first and second cavities, respectively. The reflectivity, $r_{2}$ determines the coupling strength between the two cavities. When the second cavity is coupled to the first cavity under the condition $\Delta v_{12}=v_{2}-v_{1}=0$, where $v_{1}$ and $v_{2}$ are resonance frequencies of the first and second cavities, respectively, a sharp transmission peak appears at $v_{2}$ in the otherwise opaque spectral region, exhibiting all-optical analogue of EIT [7]. As the laser frequency is scanned across the resonance, the reflected phase jump in Eq. (1) is $4 \pi \mathrm{rad}$ under the condition $r_{1}<\rho_{1}$. This value is contrast to that of the single resonator, i.e. $\pi$ and $2 \pi \mathrm{rad}$ in the transmission and reflection configurations, respectively [11], and indicates that we can improve the delay-bandwidth product in the coupled resonator over a single resonator.

A frequency tunable 532-nm continuous wave from $\mathrm{Nd}^{3+}$ :YAG laser with a linewidth of 1 $\mathrm{kHz}$ was used as the light source and mode-hop-free tuning range was over $10 \mathrm{GHz}$. Transformlimited Gaussian temporal pulses of $t_{p}=46 \mathrm{~ns}$ were generated using an electro-optic modulator. The laser beam was expanded using two lenses, and an amplitude mask in the shape of a "Y" was inserted into the collimated beam. A neutral density filter of optical density of 0.1 was inserted in the first cavity in order to achieve a nearly critical coupling condition. The detuning between the first and second cavities, $\Delta v_{12}$, was controlled by tuning the cavity length of the second cavity. The 
reflected images were detected using a cooled charge-coupled device camera and the temporal profile of images was measured using a streak camera.

Figure 2(a) and 2(b) show the reflection spectra from the coupled image resonator as a function of the laser frequency $v$ under the condition $\Delta v_{12}=0$. A narrow transmission peak arose at $\delta v=v-v_{2}=0$ owing to destructive interference between the two resonators, exhibiting coupledresonator-induced transparency. As we increase the coupling by reducing the value of $R_{2}$ from 0.9 to 0.8 , the width of the induced window increases as is seen from Figs. 2 (a) to 2(b). The relevant dispersion curve also changes, hence, the tunable delay time proportional to inverse of the band width is expected. In this context, the delay time is calculated using Eq. (1) and the parameters used in Fig. 2 (a) and 2(b) which are $\tau_{c a l}=9.0 \mathrm{~ns}$ and $\tau_{c a l}=5.7 \mathrm{~ns}$, respectively. Figure 2(c) and 2(d) show the reflection spectra under the conditions, $\Delta v_{12} \neq 0$. As a characteristic feature of this offresonance effect, the induced transmission peak reshapes into sharp asymmetric structures, which are attributed to Fano effect [12]. Figure 2(e) shows the experimental observation of two-dimensional optical images propagated through the coupled image resonator at the four frequencies $A, B, C$, and $D$ as shown in Fig. 2(a). Image (A) is at the off-resonance frequency, images (B) and (D) are at the lower and higher frequency sides of the transparency window, respectively, where the transmission minima occur, and image (C) is at the center of the induced transparency window. The overall images were well propagated through the coupled image resonator, although the edge regions of the images were distorted due to diffraction. The fact that the coupled image resonator is capable of transmitting images is crucial, allowing delayed images via the induced transparency.

Figure 3 shows temporal profiles of the images propagated through the coupled resonator, both at the off-resonance and at the induced transparency window. The pulse peak observed at the center frequency of the induced transparency was delayed by $\tau_{\text {exp }}=10.6 \mathrm{~ns}$. This value shows good agreement with the above mentioned group delay time, $\tau_{c a l}=9.0 \mathrm{~ns}$, calculated using Eq. (1). The 
experimental results, therefore, demonstrate that the two-dimensional optical images are propagated through the coupled-resonator-induced-transparency window with a large delay, and without significant attenuation, amplification, or pulse deformation.

To analyze the spatial deformation of the image due to diffraction, we define the spatial and temporal bandwidth of the amplitude transfer function, $T\left(k_{x}, k_{y}, v\right)$, as $\Delta k$, and $\Delta v$, respectively. Using Eq. (1), these bandwidths were calculated as $\Delta v=46 \mathrm{MHz}$ and $\Delta k=6.9 \times 10^{3} \mathrm{~m}^{-1}$. The bandwidths of the injected image were $\delta v=0.441 / t_{p}=9.6 \mathrm{MHz}$, where $t_{p}=46 \mathrm{~ns}$, and $\delta k=3.1 \times 10^{3} \mathrm{~m}^{-1}$. Therefore, the main spatial and temporal Fourier components of the image lied within the bandwidth of the image resonator used. This means that the spatial and temporal profiles did not undergo significant deformation or pulse broadening. Under conditions, $\Delta v_{12} \neq 0$ (Figs. 2 (c) and (d)), spectral structure exhibits sharp asymmetry, which also influences the amplitude transfer function in $k$-space. When $\Delta v_{12}>0$, the transmission peak tends to have a tail toward the lower frequency side, and this asymmetry increases the relevant $k$-space bandwidth. Using the parameters used in Fig. 2(c), the spatial bandwidth can be increased as $\Delta k=9.1 \times 10^{3} \mathrm{~m}^{-1}$. While the observed delay in the present system is of the order of input pulse duration, it may be possible to lengthen the delay using a periodic array of coupled resonators. Such a system can be considered as a one-dimensional photonic crystal; and the results and the advantages of one dimensional photonic crystal may be applicable $[13,14]$. The uniqueness of coupled resonator system may be used to break the delay-bandwidth limit via dynamic tuning of the individual coupled cavities [6].

In conclusion, we have experimentally demonstrated the ability to propagate transverse twodimensional slow images through a coupled-resonator-induced transparency and also have described the development in achieving all-optical tunable image delay. The significance of coupled resonator system is that it is free from fundamental drawback such as atomic diffusion, though efforts are necessary for the complete elimination of diffraction effect on slow images [15]. The present 
coupled resonator system used for the study is stable to observe the image propagation. However, more sophisticated technologies, such as modern etalon manufacturing techniques may be useful in practical applications. Alternative promising photonic structures may be bundled fiber optic image plate, in which fiber Bragg gratings can be implemented with the goal of diffraction free all-optical methods for delaying images. These photonic structures could have potential to practical image applications such as in holography to optically store, retrieve and process information. 


\section{References}

1. Focus Issue: "Slow light," Nature Photon. 2, 447-509 (2008).

2. J. B. Khurgin, R. S. Tucker, Slow Light: Science and Applications (CRC Press, 2008)

3. J. K. S. Poon, J. Scheuer, Y. Xu, and A. Yariv, "Designing coupled-resonator optical waveguide delay lines,” J. Opt. Soc. Am. B 21, 1665-1673 (2004).

4. M. S. Shahriar, G. S. Pati, R. Tripati, V. Gopal et al, "Ultrahigh enhancement in absolute and relative rotation sensing using fast and slow light,” Phys. Rev. A 75, 053807 (2007).

5. M. F. Yanik and S. Fan, “Stopping light all optically,” Phys. Rev. Lett. 92, 083901 (2004).

6. Q. Xu, P. Dong, and M. Lipson, "Breaking the delay-bandwidth limit in a photonic structure, "Nature Physics, 3, 406 (2007).

7. K. Totsuka, N. Kobayashi, and M. Tomita, "Slow light in coupled resonator induced transparency," Phys. Rev. Lett. 98, 213904 (2007).

8. R. M. Camacho, C. J. Broadbent, I. Ali-Khan, and J. C. Howell, “All optical delay of images using slow light,” Phys. Rev. Lett. 98, 043902 (2007).

9. P. K. Vudyasetsu, R. M. Camacho, and J. C. Howell, "Storage and retrieval of multimode transverse images in hot atomic rubidium vapor,” Phys. Rev. Lett. 100, 123903 (2008).

10. M. Shuker, O. Firstenberg, R. Pugatch, A. Ron, and N. Davidson, "Storing images in warm atomic vapor,” Phys. Rev. Lett. 100, 223601 (2008).

11. M. Tomita, P. Sultana, A. Takami, and T. Matsumoto, "Advanced and delayed images through an image resonator,” Opt. Express. 18, 12599 (2010).

12. M. Tomita, K. Totsuka, R. Hanamura, and T. Matsumoto "Tunable Fano interference effect in coupled-microsphere resonator-induced transparency”, J. Opt. Soc. Am. B 26, 813 (2009)

13. S. Molchanov and B. Vainberg, "Slowdown of the wave packages in finite slabs of periodic media,” Waves Random Media 14, 411 (2004).

14. A. Figotin and I. Vitebskiy, "Slow light in photonic crystals," Waves in Random and Complex Media. 16, 293 (2006).

15. O. Firstenberg, M. Shuker, N. Davison, and A. Ron, "Elimination of the diffraction of arbitrary images imprinted on slow light,” Phys. Rev. Lett. 102, 043601 (2009). 


\section{Figure Captions}

Fig. 1 Schematic illustration of image propagation through coupled image resonator in the reflection geometry. $\mathrm{M}$ is the mirror, $\mathrm{HM}$ is the half mirror, and $\mathrm{N}$ is the neutral density filter.

Fig. 2 (a)-(d) Experimental observation of the reflection spectra from the coupled image resonator. The reflectance of the three mirrors are $\mathrm{R}_{1}=0.5 ; \mathrm{R}_{2}=0.9$ in (a), (c) and (d), while $\mathrm{R}_{2}=0.8$ in (b); $\mathrm{R}_{3}=0.99$. The detuning were $\Delta v_{12}=0$ in (a) and (b), $\Delta v_{12}=119 \mathrm{MHz}$ in (c) $\Delta v_{12}=238 \mathrm{MHz}$ in (d). The cavity lengths are $\mathrm{L}_{1}=10 \mathrm{~cm}$ and $\mathrm{L}_{2}=20 \mathrm{~cm}$. Solid lines show the experimental observation, dotted solid lines show the theoretical calculation based on Eq. (1). 2 (e) is the experimental observation of reflected images. (A), (B), (C), and (D) are at frequencies $A, B, C$, and $D$ indicated in Fig. 2 (a).

Fig. 3 Experimental observation of temporal profile of the reflected image. The dashed and solid curves are the pulse profiles at two laser detuning frequencies, $A$ and $C$, shown in Fig. 2(a). The origin of the time axis, i.e., $\tau=0$, is taken at the pulse peak observed at the off-resonance frequency. 
Fig-1

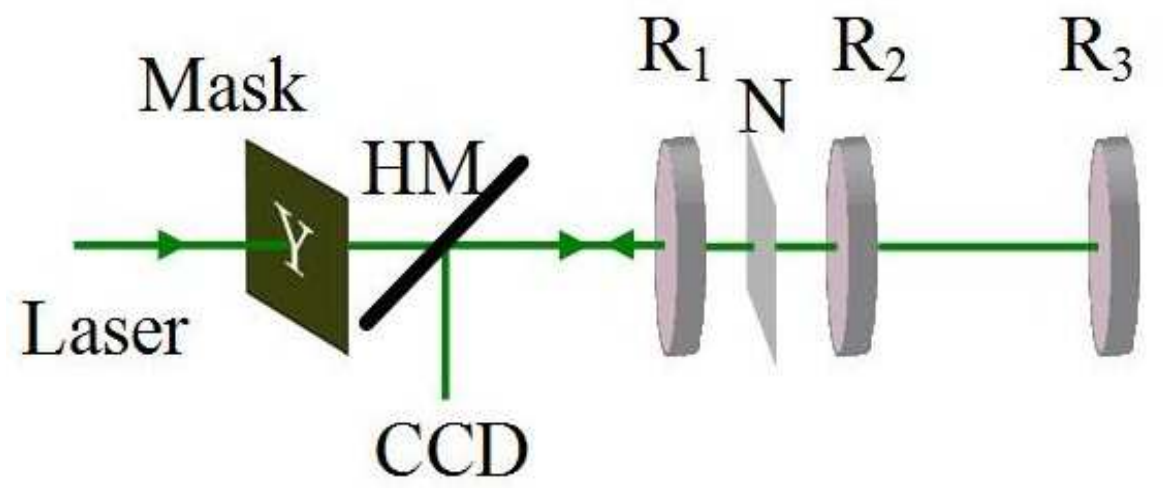


Fig-2
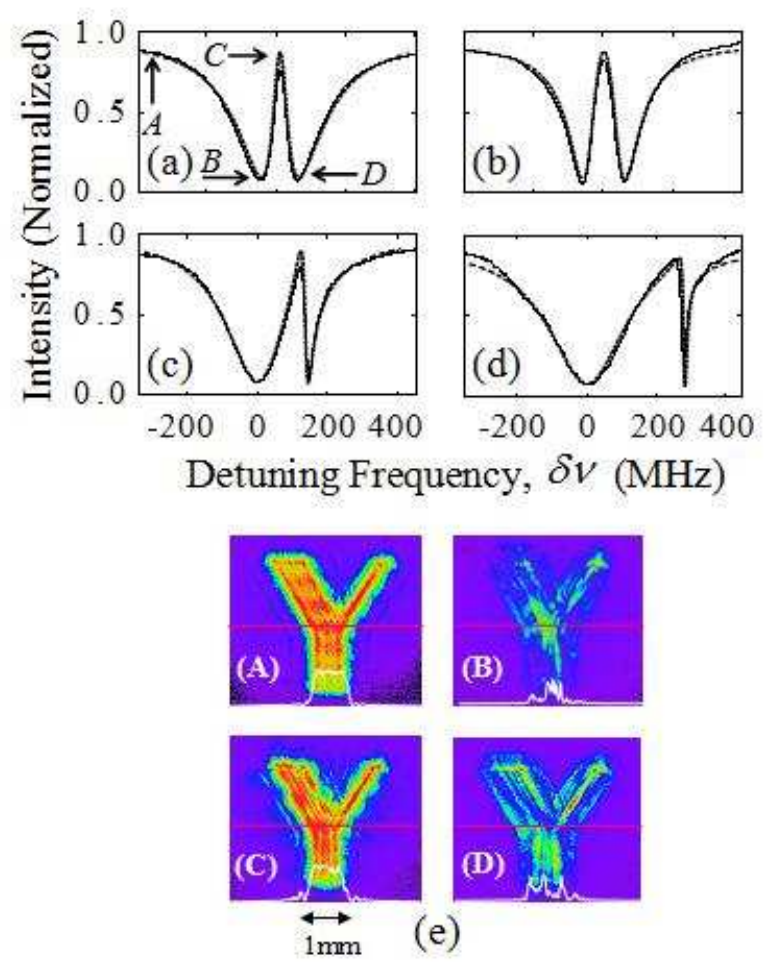
Fig-3

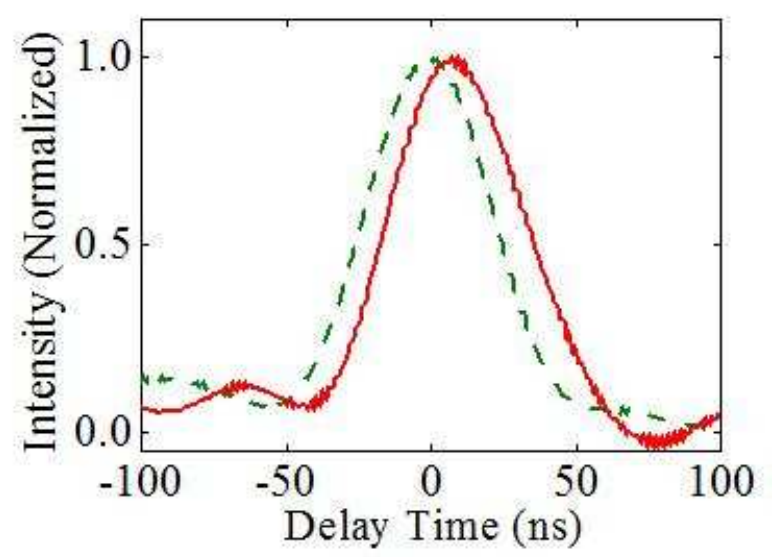

